\title{
Evidence for prolonged main sequence stellar evolution of $F$ stars in close binaries ${ }^{\star}$
}

\author{
A. A. Suchkov ${ }^{\star \star}$ \\ Space Telescope Science Institute, NASA, Baltimore, MD 21218, USA \\ Received 25 July 2000 / Accepted 22 January 2001

\begin{abstract}
Binary F stars exhibit large brightness anomaly, which is defined here as the difference between the absolute magnitude from the uvby photometry and the actual absolute magnitude of the star. We have found that the anomaly inversely correlates with the binary components separation. There is evidence that the correlation reflects actual population differences between close and wide binary pairs, in which case it indicates that the anomaly is somehow associated with the interaction of binary's components. The anomaly has also been found to correlate with both kinematics and metallicity. The sense of the correlations implies that the anomaly increases as the star evolves, suggesting a peculiar evolution of a primary F star in a tight binary pair. This conclusion has further been supported by the study of the age-velocity relation (AVR) of F stars that are cataloged in the HIPPARCOS as single. Among these stars, those with brightness anomaly were previously shown to be most likely unidentified close binaries. We have found that the AVR of these binary candidates is different from that of the "truly single" F stars. The discrepancy between the two AVRs indicates that the putative binaries are, on average, older than similar normal single F stars at the same effective temperature and luminosity, which is consistent with the inferred peculiar evolution in close binaries. It appears that this peculiarity is caused by the impact of the components interaction in a tight pair on stellar evolution, which results in the prolonged main sequence lifetime of the primary $\mathrm{F}$ star.
\end{abstract}

Key words. binaries: close - stars: evolution

\section{Introduction}

Many of the F stars cataloged in the HIPPARCOS as single were recently argued to be in fact unidentified binaries (Suchkov \& McMaster 1999). The criterion used to isolate binary candidates involves the difference between the absolute magnitude $M_{V}$ based on the HIPPARCOS parallax and the absolute magnitude $M_{c_{0}}$ derived from the uvby luminosity index $\Delta c_{0}$, i.e., $\Delta M_{c_{0}}=M_{c_{0}}-M_{V}$. For the best known nearby single $\mathrm{F}$ stars $(d<25 \mathrm{pc})$, the two magnitudes coincide within \pm 0.15 mag. However, most of the unresolved binary stars are anomalously bright for their uvby colors, i.e., their absolute magnitude, $M_{V}$, is substantially brighter than the magnitude derived from $\Delta_{c_{0}}: \Delta M_{c_{0}}>0.15$ (for simplicity, we will often be referring to $\Delta M_{c_{0}}$ as brightness anomaly and consider a star as anomalously bright if $\left.\Delta M_{c_{0}}>0.15\right)$. Unlike the nearby single stars, many of the distant single F stars, $(d>25 \mathrm{pc})$ turned out to be anomalously bright as well, similar to the known binary $\mathrm{F}$ stars. The analysis showed that in this case, the brightness anomaly is likely associated with the

\footnotetext{
* Based on the data from the Hipparcos astrometry satellite (European Space Agency).

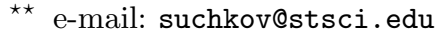

presence of undetected companions (Suchkov \& McMaster 1999). These binary candidates were called $\mathrm{C}$ binaries (the follow-up radial-velocity survey of northern $\mathrm{C}$ binaries with $\Delta M_{c_{0}} \geq 0.5$ and $d<80$ pc conducted by Griffin \& Suchkov 2001 has already confirmed that more than $35 \%$ of the sample stars actually are binaries; orbits for many of these binaries have been determined).

For unresolved binaries, $M_{c_{0}}$ is derived from the color indices originating from a double star combined spectrum. Because of that $\Delta M_{c_{0}}$ underestimates the luminosity of that star if its components are comparably bright, by up to $-2.5 \log 2 \approx-0.75 \mathrm{mag}$ in the case of identical components (this obviously imposes an upper limit of 0.75 on $\left.\Delta M_{c_{0}}\right)$. However, this seems to be not the only, and perhaps not even the main effect leading to the discrepancy between $M_{c_{0}}$ and $M_{V}$. For one thing, a substantial fraction of binary stars have brightness anomaly well above the indicated upper limit of 0.75 mag. The stars with $\Delta M_{c_{0}}>0.75$, which cannot be explained in terms of the combined flux of the unresolved binary components, indicate that along with the combined luminosity something else may impact the absolute magnitude of unresolved binary stars. 
More evidence favoring the existence of an additional source for brightness anomaly has come from the study of the relationship between $\Delta M_{c_{0}}$ and stellar age (Suchkov 2000). It turns out that in terms of kinematics and metallicity, anomalously bright binary candidates, $\mathrm{C}$ binaries, are, on average, substantially older than the "truly single" F stars, i.e., the stars with normal brightness. If the anomaly were caused entirely by the luminosity contribution from the undetected secondary, one would have to conclude that binaries with the components having about the same mass live longer or tend to form earlier in the galactic evolution than binaries with disparate components. But such a scenario does not seem very likely. A more plausible interpretation of the relationship between $\Delta M_{c_{0}}$ and age might be that at least some part of the star's brightness anomaly is associated with a peculiar stellar evolution in the binary system, so that the primary $\mathrm{F}$ star in that binary stay within the main sequence longer than a normal single F star. Given the obviously important implications of such a possibility, we have examined a number of relationships between $\Delta M_{c_{0}}$ and other parameters of $\mathrm{F}$ stars in order to get a deeper insight into the nature of brightness anomaly.

\section{Data}

The star sample used in this study originates from the list of $\sim 10000$ HIPPARCOS stars that have measured uvby colors, with effective temperatures corresponding to the spectral range occupied by $\mathrm{F}$ and early $\mathrm{G}$ stars (Suchkov \& McMaster 1999). The uvby data are from Hauck \& Mermilliod (1998). We have computed tangential velocities of these stars from the HIPPARCOS parallaxes and proper motions. The HIPPARCOS parallax has also been utilized to derive absolute magnitude $M_{V}$ from the Johnson $V$ magnitude and absolute magnitude $M_{H p}$ from the HIPPARCOS Hp magnitude. Absolute magnitude $M_{c_{0}}$ has been calculated from the uvby data with the algorithm published by Moon (1985; see also Moon \& Dworetsky 1985); these calculations involve the dereddened uvby luminosity index, $\Delta c_{0}$, along with the dereddened metallicity and temperature indices. The algorithm in Moon (1985) has also been used to compute standard $\beta$ from the blanketing-corrected color index $(b-y)_{\mathrm{cor}}$, from which effective temperature has been obtained.

Metallicity has been calculated from the dereddened uvby color indices, utilizing the calibration given by Schuster \& Nissen (1989) for F stars. This calibration is somewhat different from that in Carlberg et al. (1985), which was used in Suchkov (2000) and Suchkov \& McMaster (1999). This difference, however, has been found not to impact the results of both the previous and present studies. The Schuster \& Nissen (1989) calibration is commonly utilized in current studies, so we will use it in this paper.

Age estimates are based on isochrone fitting in the $\log T_{\mathrm{e}}-M_{V}$ diagram using Yale (1996) isochrones

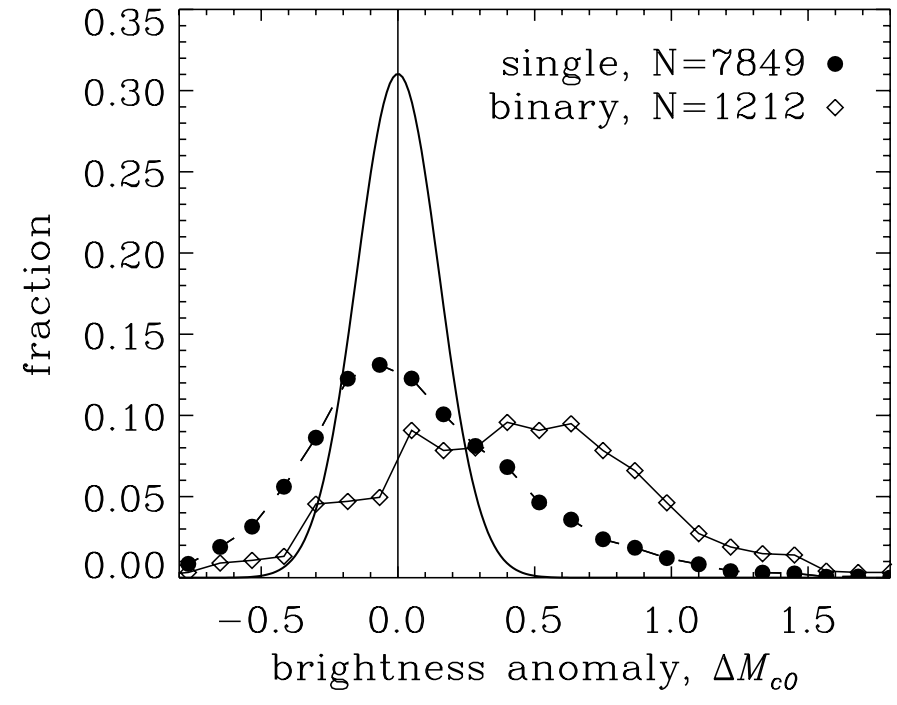

Fig. 1. Normalized brightness anomaly distribution for the unresolved binaries and the stars cataloged in the HIPPARCOS as single. The Gaussian is for the parameters derived from the sample of single F stars within $25 \mathrm{pc}$

(Demarque et al. 1996 ${ }^{1}$ ). Age has been used only in terms of mean values obtained by averaging over large groups of stars, and only in differential analysis involving relative ages. Therefore, age accuracy, both in terms of random and systematic errors, is not of much concern for the present study.

The sample has been constrained as follows: $0.22 \leq$ $(b-y) \leq 0.39 ; 5800 \leq T_{\mathrm{e}} \leq 7500, \mathrm{~K} ;-0.6 \leq[\mathrm{Fe} / \mathrm{H}] \leq 0.5$; $0.02 \leq e_{c 1}\left(e_{c 1}\right.$ is the mean error on $c_{1}$ for an individual star as given in Hauck \& Mermilliod 1998; the sample mean for $e_{c 1}$ is $\sim 10$ times smaller); $0.015 \leq e_{m 1}\left(e_{m 1}\right.$ is the mean error on $m_{1}$ for an individual star); $3 \geq e_{\pi}$, mas (error on parallax); $3 \geq e_{\mu_{\alpha}}$, mas $\mathrm{yr}^{-1}$ (error on proper motion in RA); $3 \geq e_{\mu_{\delta}}$, mas $\mathrm{yr}^{-1}$ (error on proper motion in DEC).

\section{Results and discussion}

\subsection{Brightness anomaly distribution}

Brightness anomaly is very conspicuous in unresolved binary stars (Fig. 1). As shown in Suchkov \& McMaster (1999), the distribution of $\Delta M_{c_{0}}$ for the best studied single stars within $25 \mathrm{pc}$ is centered at $\Delta M_{c_{0}}=0$ and is rather narrow, with standard deviation of only 0.15 mag. The corresponding Gaussian $\left(\sigma_{\Delta M_{c_{0}}}=0.15\right)$ is shown in Fig. 1. For the unresolved binaries, the maximum of the $\Delta M_{c_{0}}$ distribution is not only offset from zero by $\sim 0.5 \mathrm{mag}$ but is also much broader. Within $200 \mathrm{pc}$, the stars cataloged in the HIPPARCOS as single have a broad distribution as well, but with the maximum only slightly shifted ( -0.1 mag) from that of the single stars within $25 \mathrm{pc}$.

\footnotetext{
1 Available at http://shemesh.gsfc.nasa.gov/astronomy.html
} 
Note that the left tail of the distribution, albeit resembling a segment of a Gaussian, is far above the level suggested by $\sigma_{\Delta M_{c_{0}}}=0.15$, meaning that it does probably not represent the error distribution for $\Delta M_{c_{0}}$. Therefore, the population of anomalously faint stars, $\Delta M_{c_{0}}<0.15$, should be as real as that of anomalously bright stars. As seen below, its distinctive identity is reflected in lowest velocities and highest metallicities among the F stars, which makes it the youngest group of $\mathrm{F}$ stars. Therefore, we conclude that in general the distributions of both binary and single stars in Fig. 1 are most likely dominated by some stellar population physics rather than random or systematic errors.

The most striking feature in Fig. 1 is the positive brightness anomaly of binary stars. Therefore, in the rest of the paper, we will focus primarily on the nature of anomalously bright binary stars.

\subsection{Correlation between $\Delta M_{c_{0}}$ and binary components separation}

If the combined flux of unresolved binary components is not the only reason for the enhanced brightness of a binary star, and there is a noticeable contribution from a physical anomaly in the binary's primary star caused by its interaction with the companion, one may expect the brightness anomaly to depend on binary components separation, because the effect of the interaction, whatever it is, is probably stronger in tighter pairs. We have tested this hypothesis by correlating brightness anomaly and projected separation for two very different, non-overlapping samples of binary $\mathrm{F}$ stars within $125 \mathrm{pc}$, for which the HIPPARCOS provides angular separation, $\rho$. Only the stars with $\Delta M_{c_{0}}$ in the range -0.5 to 1.0 have been included, so that the potentially less reliable extreme values of $\Delta M_{c_{0}}$ do not impact the results. The chosen distance limit rejects the most distant stars, thus ensuring better data quality for the remainder of the sample; at the same time it retains enough stars for statistical tests we are interested in.

The stars in the first sample are binaries discovered by HIPPARCOS (see Mignard et al. 1992; Söderhjelm et al. 1992 for the procedures to detect and measure binary stars on the basis of HIPPARCOS observations). Only the binaries with angular separation $\rho \leq 1$ arcsec have been included; this constraint rejects only a very small fraction of the HIPPARCOS binaries and is not of great significance for the test below. The sample has also been constrained with respect to angular separation error, $\epsilon_{\rho}$, to include only the stars with $\epsilon_{\rho} / \rho<0.1$.

The second sample comprises unresolved binaries known prior to HIPPARCOS. With respect to angular separations, it has been constrained similar to the sample of the HIPPARCOS binaries, $\rho \leq 1$ arcsec. The preHIPPARCOS binaries are much more numerous, which allows us to impose a more stringent constraint on the angular separation accuracy: $\epsilon_{\rho} / \rho<0.01$. Additionally, this

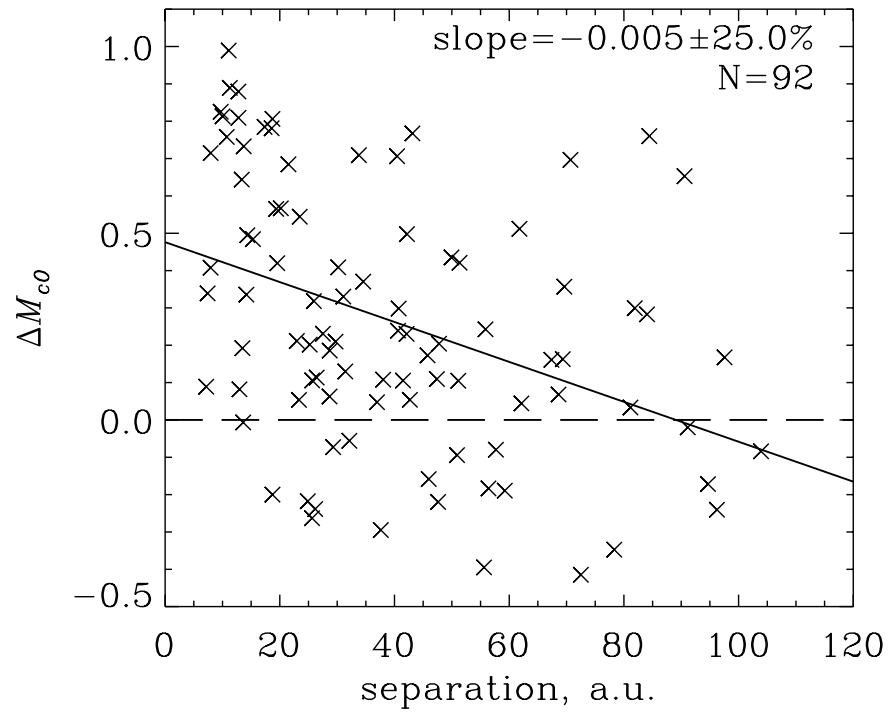

Fig. 2. Correlation between brightness anomaly and binary components separation for a distance limited sample $(d \leq$ $125 \mathrm{pc}$ ) of binary $\mathrm{F}$ stars discovered by HIPPARCOS. The sample stars have angular separation within 1 arcsec

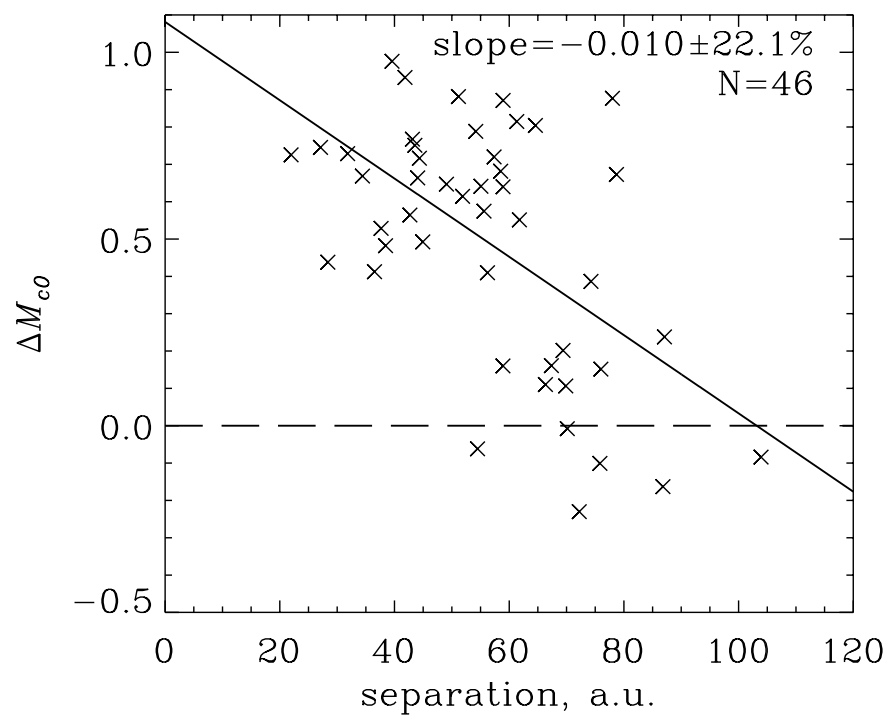

Fig. 3. Correlation between brightness anomaly and binary components separation for unresolved pre-HIPPARCOS binary F stars within $125 \mathrm{pc}$ with angular separation $\rho \leq 1.0 \operatorname{arcsec}$

sample has been constrained to include only hot stars, $6500-7500 \mathrm{~K}$. This should improve chances to detect any separation-dependent evolutionary effects in $\Delta M_{c_{0}}$ just because the fraction of evolved stars increases toward higher temperatures (at cooler temperatures, brightness anomaly in the sample stars may be dominated by the separation-independent effect of the combined flux of binary's unresolved components).

Projected separation for both samples has been derived from the parallax, $\pi$, and the angular separation, $\rho$, given in the HIPPARCOS catalogue.

The stars of the two samples are presented in Figs. 2 and 3. As one can see, they do indeed show a correlation between separation and brightness anomaly, with the 


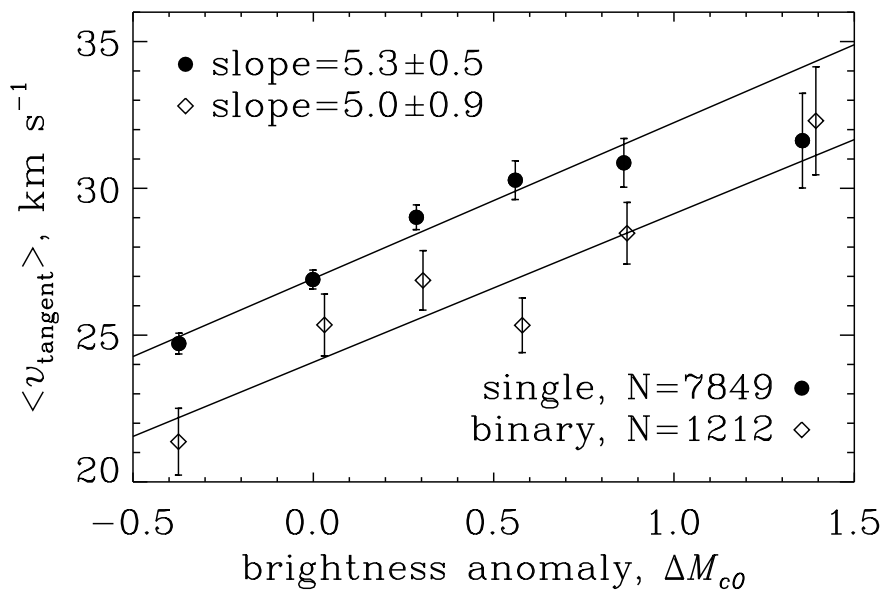

Fig. 4. Mean tangential velocity versus $\Delta M_{c_{0}}$ for the unresolved binary $\mathrm{F}$ stars and $\mathrm{F}$ stars cataloged in the HIPPARCOS as single. Weighted linear regression and its slope are shown for both groups of stars

slope of the linear regression being non-zero well above the error in both cases (the slope error is given as a percent of the slope value).

It is to be noted that the correlation remains the same when absolute magnitude $M_{V}$ in the definition of $\Delta M_{c_{0}}$ is replaced with absolute magnitude $M_{H p}$ obtained from the HIPPARCOS Hp magnitude (for the details of HIPPARCOS photometry see Mignard et al. 1992; Evans et al. 1992). The corresponding regression slopes are: $-0.005 \pm 25.2 \%$ for the HIPPARCOS binaries, and $-0.011 \pm 21.6 \%$ for the pre-HIPPARCOS binaries.

The correlation in Figs. 2 and 3 reveals that $\Delta M_{c_{0}}$ is, on average, larger at smaller component separations. This suggests that brightness anomaly is somehow associated with the components interaction in a tight binary pair. The question is then: what is the physics behind this anomaly?

\subsection{Relationship between $\Delta M_{c_{0}}$ and stellar kinematics}

To answer the above question, we have explored whether there is any dependence in $\Delta M_{c_{0}}$ on age, because the effects caused by interaction in a close binary may accumulate with time. First of all, we have correlated $\Delta M_{c_{0}}$ and tangential velocity, employing the fact that the statistics from stellar kinematics, such as mean tangential velocity and velocity dispersion used here, are age-sensitive.

As seen in Fig. 4, there is indeed a significant correlation between $\left\langle v_{\text {tangent }}\right\rangle$ and $\Delta M_{c_{0}}$. It implies that $\Delta M_{c_{0}}$ is larger, on average, at older ages. Given that the $H I P P A R C O S$ single stars with large $\Delta M_{V c_{0}}$ are probable close binaries, we conclude that this result is consistent with increase in brightness anomaly for a tight binary pair as the primary star evolves.

Figure 4 also reveals that the kinematics of known binary stars is "younger" than that of the single stars at the same $\Delta M_{c_{0}}$. This reflects the generally younger age of the population of the known binary F stars, which was discussed in Suchkov (2000).

\subsection{Relationship between $\Delta M_{c_{0}}$ and metallicity}

Mean metallicity is another age-dependent statistics that can be used to check if there is any relationship between $\Delta M_{c_{0}}$ and age. The correlation between $[\mathrm{Fe} / \mathrm{H}]$ and $\Delta M_{c_{0}}$ is shown in Fig. 5 for the same groups of stars as in Fig. 4. Here, the linear regression slope is non-zero within more than one sigma for both the binary and single star samples, indicating that $\Delta M_{c_{0}}$ is larger at lower metallicities. Since lower metallicity is indicative of older age, the correlation in Fig. 5 is consistent with the inference from the preceding subsection that the stars with larger $\Delta M_{c_{0}}$ are, on average, older.

The correlation in Fig. 5 could have been expected on the basis of the correlation in Fig. 4 because metallicity is known to correlate with kinematics. At the same time this correlation is obviously weaker than that in Fig. 4. Since metallicity is also known to have a large spread at any given age, a possible interpretation of weaker correlation may be that metallicity is coupled with age more loosely than kinematics.

The weaker correlation in Fig. 5, whatever its reason, argues against the possibility that the correlation in Fig. 4 results from a metallicity-dependent bias in estimates of $M_{c_{0}}$, hence $\Delta M_{c_{0}}$. If such a bias did exist (say, due to flaws in the algorithm that computes $M_{c_{0}}$ ) and cause all the correlation in Fig. 5, one would have expected it to translate into a weaker rather than stronger correlation between $\Delta M_{c_{0}}$ and kinematics in Fig. 4 . It is to be added that the aforementioned hypothetical bias seems unlikely anyway, because the available evidence, including the results from the binary components separation data and the age-velocity relation (see below), argues against any role of this kind of systematic errors in brightness anomaly.

Thus, the interpretation of both Figs. 4 and 5 involving the dependence of brightness anomaly on age appears to be reasonably justified.

\subsection{Age-velocity relation: Discrepancy between normal and anomalously bright stars}

So far, we have been referring to brightness anomaly in the sense that the absolute magnitude of a star with this anomaly is brighter than that of a normal single star with the same uvby colors. But it would be equally correct to say that the star has the uvby colors that are anomalous for a normal star at a given effective temperature and luminosity. The anomaly in this case refers to the color index $c_{0}$.

For the main sequence $\mathrm{F}$ stars, $c_{0}$ provides a measure of Balmer discontinuity (see Crawford 1975 and references therein). In these stars, smaller Balmer discontinuity at a given effective temperature (hence smaller $c_{0}$ ) means a denser stellar atmosphere, i.e., larger surface gravity. In 


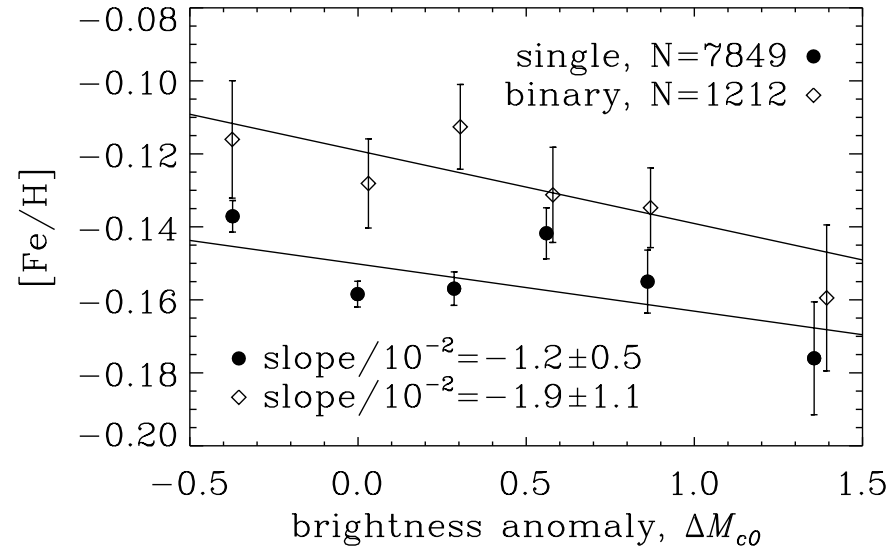

Fig. 5. Metallicity versus $\Delta M_{c_{0}}$ for the unresolved binary $\mathrm{F}$ stars and $\mathrm{F}$ stars cataloged in the HIPPARCOS as single. Weighted linear regression and its slope are shown for both groups of stars

this way, $c_{0}$ actually measures stellar surface gravity (at a given $T_{\mathrm{e}}$ ), through which it is related to the star's luminosity. Anomalous $c_{0}$ in an F star then means anomalous surface gravity, $\log g$. Specifically, the anomaly resulting in a positive $\Delta M_{c_{0}}$ corresponds to the surface gravity that is too high for a given effective temperature, $T_{\mathrm{e}}$, and luminosity, $L$. Consequently, the stellar mass of such a star is larger than the mass of a normal single star at the same $T_{\mathrm{e}}$ and $L$. (Alternatively, one can say that the luminosity of an anomalously bright star is too high for a given $T_{\mathrm{e}}$ and $\log g$.) This obviously requires that stellar evolution of a normal star is different from that of an anomalously bright star. So, if $c_{0}$ of the anomalous stars in our sample is dominated by the effect of enhanced surface gravity, these stars can be predicted to evolve in a non-standard way.

We can verify the latter prediction as follows. Let us take the sample of the HIPPARCOS "single" stars and split it into two subsamples: the first one including only anomalously bright stars, $\Delta M_{c_{0}}>0.15$ (C binaries), and the second subsample that includes the stars with $\Delta M_{c_{0}}<0.15$; for simplicity, the latter stars will be referred to as "truly single", although we expect a fraction of them to be unevolved and/or wide binaries with disparate components. Now, we can derive and compare the age-velocity relations (AVR) for these two group of stars. If the same stellar evolution model (meaning the same set of isochrones used to derive age) is applicable to all stars, we should obviously get the same AVR for both groups, simply because stellar kinematics and stellar evolution are entirely unrelated things. If this proves not to be the case, and the age-velocity relations are discrepant, the two groups of stars evolve probably differently.

We have derived ages for both groups of stars based on isochrones from Demarque et al. (1996). The isochrones as well as the stars used to calculate the age-velocity relations are shown in Fig. 6. The stars have been selected in a narrow metallicity range, $-0.3<[\mathrm{Fe} / \mathrm{H}]<-0.1$, that matches the chemical composition of the isochrones,

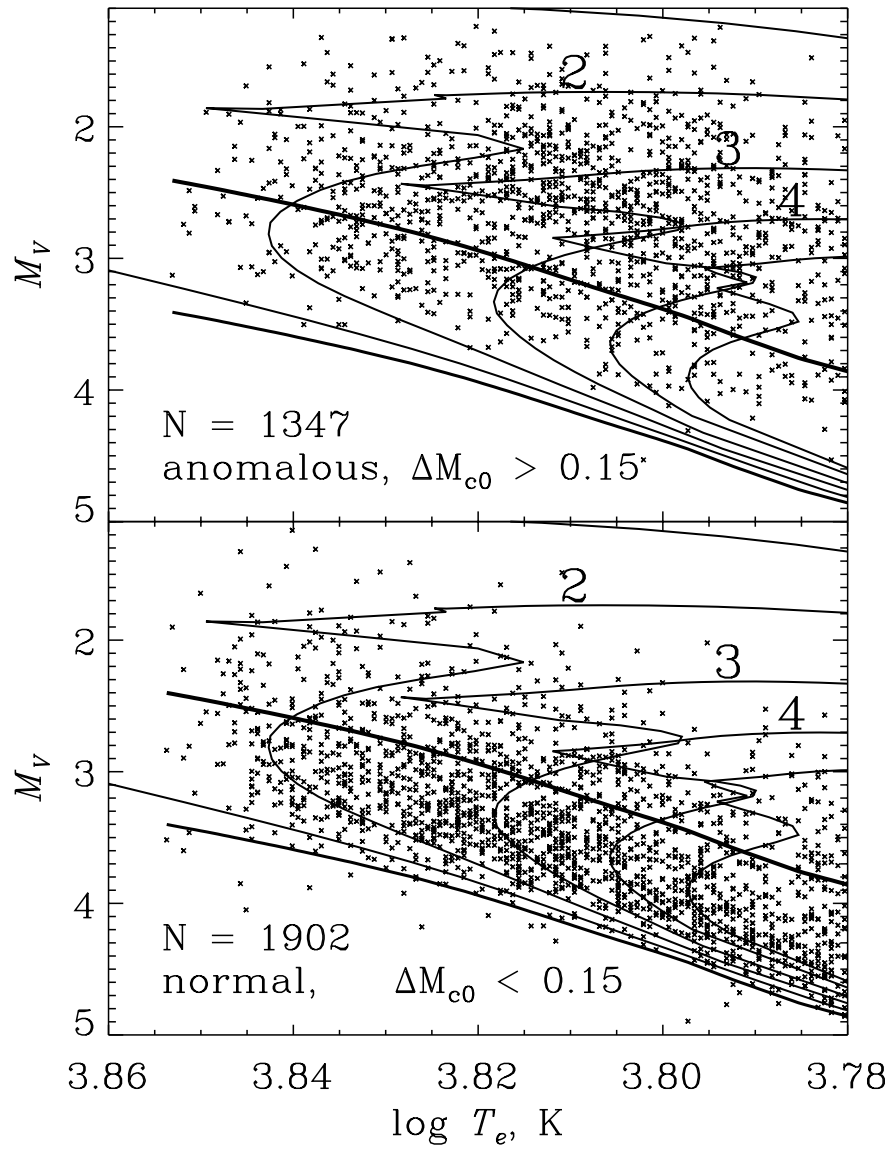

Fig. 6. $\log T_{\mathrm{e}}-M_{V}$ diagram of the HIPPARCOS "single" F stars. Upper panel: anomalously bright stars, presumably dominated by unidentified close binaries ( $\mathrm{C}$ binaries). Lower panel: stars with mostly normal brightness, presumably comprising truly single stars as well as unevolved close binaries with disparate components. The isochrones are labeled by their age in Gyr. The level of 1.0 mag above ZAMS is shown (see text for details)

$Z=1.010^{-2}, \quad Y=0.27$. Additionally they have been constrained to the distance range $d \leq 125 \mathrm{pc}$. They have been grouped into 1 to 2 Gyr age bins, for which mean tangential velocity has been calculated. Because of large age errors for the young stars near the ZAMS, ages younger 1 Gyr have not been considered. The resulting age-velocity relations for the age range from 1 to $8 \mathrm{Gyr}$ are given in Fig. 7.

Figure 7 reveals a conspicuous discrepancy between the two age-velocity relations, which will be referred to as the AVR discrepancy. The possibility that kinematic differences between the normal and anomalously bright stars arise from different distance sampling (the latter stars are, on average, more distant) was checked and ruled out in Suchkov (2000). We have also looked into the possibility that the bias associated with the brighter, on average, absolute magnitudes of anomalously bright stars (see Fig. 6) plays a role in the discrepancy (for example, this may happen if the isochrones underestimate the age of far evolved stars). To this end, we have compared the 
Table 1. Kinematics of normal ("truly single") and anomalously bright (C binaries ) F stars above and below the line $M_{\mathrm{ZAMS}}-M_{V}=1.0$ shown in Fig. 6

\begin{tabular}{lcccc}
\hline \hline brightness & $\begin{array}{c}\Delta M_{c_{0}} \\
(\mathrm{mag})\end{array}$ & $N$ & $\begin{array}{c}<v_{\text {tangent }}> \\
\left(\mathrm{km} \mathrm{s}^{-1}\right)\end{array}$ & $\begin{array}{c}\sigma_{\text {tangent }} \\
\left(\mathrm{km} \mathrm{s}^{-1}\right)\end{array}$ \\
\hline & \multicolumn{4}{c}{$M_{\text {ZAMS }}-M_{V}>1.0 \mathrm{mag}$} \\
normal $\ldots . .$. & $<0.15$ & 1226 & $26.4 \pm 0.5$ & $16.5 \pm 0.3$ \\
anomalous $\ldots$. & $>0.15$ & 2550 & $30.2 \pm 0.4$ & $18.2 \pm 0.3$ \\
& \multicolumn{4}{c}{$M_{\text {ZAMS }}-M_{V}<1.0 \mathrm{mag}$} \\
normal $\ldots . .$. & $<0.15$ & 3416 & $26.0 \pm 0.3$ & $16.6 \pm 0.2$ \\
anomalous $\ldots$. & $>0.15$ & 876 & $29.2 \pm 0.7$ & $20.9 \pm 0.5$ \\
\hline
\end{tabular}

kinematics of the normal and anomalously bright stars with $-0.6<[\mathrm{Fe} / \mathrm{H}]<0.3$ in the two regions of the $\log T_{\mathrm{e}}-M_{V}$ diagram, above and below the line corresponding to $1.0 \mathrm{mag}$ above the ZAMS shown in Fig. 6. As seen in Table 1, anomalously bright stars close to the ZAMS have velocities higher, on average, than those of the normal stars far above the ZAMS. This rules out the indicated bias as the cause of the AVR discrepancy. Therefore, the discrepancy must be real, reflecting some inconsistency in the derived ages. Assuming that the isochrones in Fig. 6 adequately represent the age of the normal stars (truly single), we must then conclude that the age of the stars with large brightness anomaly ( $\mathrm{C}$ binaries) is underestimated. The amount of the AVR discrepancy suggests that the underestimation is, on average, as large as 2 Gyr or more. Thus, anomalously bright stars are consistent with being actually older than normal stars at the same positions in the $\log T_{\mathrm{e}}-M_{V}$ diagram.

There are at least two reasons why isochrone fitting could be inadequate for anomalously bright stars and result in incorrect ages: (i) combined flux of unidentified binaries with comparably bright components, and (ii) nonstandard stellar evolution.

If anomalously bright stars are unidentified binaries with comparably bright components, isochrone fitting results in either overestimated or underestimated age, depending on the position of the star in the $\log T_{\mathrm{e}}-M_{V}$ diagram and the contribution of the secondary into the star's $V$ magnitude. For the stars cataloged in the HIPPARCOS as single, age would be, on average, underestimated (Suchkov \& McMaster 1999).

The following, however, argues against the hypothesis that such binaries dominate our sample of anomalously bright stars.

First, the sample stars are much older (on average) than the known unresolved binaries (Suchkov 2000; see also Figs. 4 and 5). This means that most of these stars are different from normal binaries. Even if they are double systems, in most cases their secondary component is hardly a regular star whose emission is comparable to that of the primary.
Second, as mentioned before, even among known binary stars a substantial fraction have brightness anomaly exceeding the maximum value of $0.75 \mathrm{mag}$, hence it cannot be attributed to the combined flux of the binary 's two components only (see Fig. 1). Statistically, the excess is quite significant, so there is little doubt that in many cases the cause of brightness anomaly is different from the light contribution from the star's unresolved companion.

So, it seems unlikely that the AVR discrepancy in Fig. 7 is due only to binaries with comparably bright components.

The above isochrone fitting can also be inadequate if for some reasons the evolution of a star is different from the normal evolution of a single star.

In such a case the position of a star in the $\log T_{\mathrm{e}}-M_{V}$ diagram would correspond to an age different from the one predicted by standard isochrones. The age-velocity relation based on ages from the standard isochrones would be incorrect for these stars, and this may be the reason for the AVR discrepancy revealed by Fig. 7 .

As discussed below, anomalous stellar evolution, unlike the effect of combined flux, offers a way to explain the relationship between age and brightness anomaly; in particular, it explains why $\mathrm{C}$ binaries are, on average, older than the truly single stars. Also anomalous evolution appears to be in line with the recent developments in studies of eclipsing binaries. This makes it a stronger candidate for being the dominant source of the AVR discrepancy in Fig. 7. The combined evidence discussed in this paper suggests then that anomalous stellar evolution occurs apparently in tight binary pairs where the evolution of the primary star is affected by the presence of the companion.

\section{Discussion and summary}

The older ages of anomalously bright stars (Suchkov 2000) seem to indicate that these stars stay within the main sequence longer than normal single stars. Such a prolonged main sequence evolution is known to occur in stars with enhanced central mixing in the convective core, and is apparently quite ubiquitous among close binary systems at stellar masses slightly above solar (see, e.g., Chiosi 1999; Andersen \& Nordström 1999; Gimenéz et al. 1999). This suggests that anomalous brightness discussed in this paper reflects non-standard stellar evolution that involves enhanced core mixing. Extreme manifestation of brightness anomaly among known unresolved binary stars seems to indicate that the anomaly is somehow related to binarity. Given that $\Delta M_{c_{0}}$ correlates with binary components separation, one may infer that interaction in a tight binary pair somehow induces extra mixing in the convective core of the binary's primary component, additional to whatever mixing occurs in a similar single star. Of course, there are other mechanisms that may impact stellar evolution in a tight binary pair. One may recall, for example, modified convection in the outer convective envelope of a star in a tight binary pair or mass loss/transfer in very close binary systems. These mechanisms were discussed in 


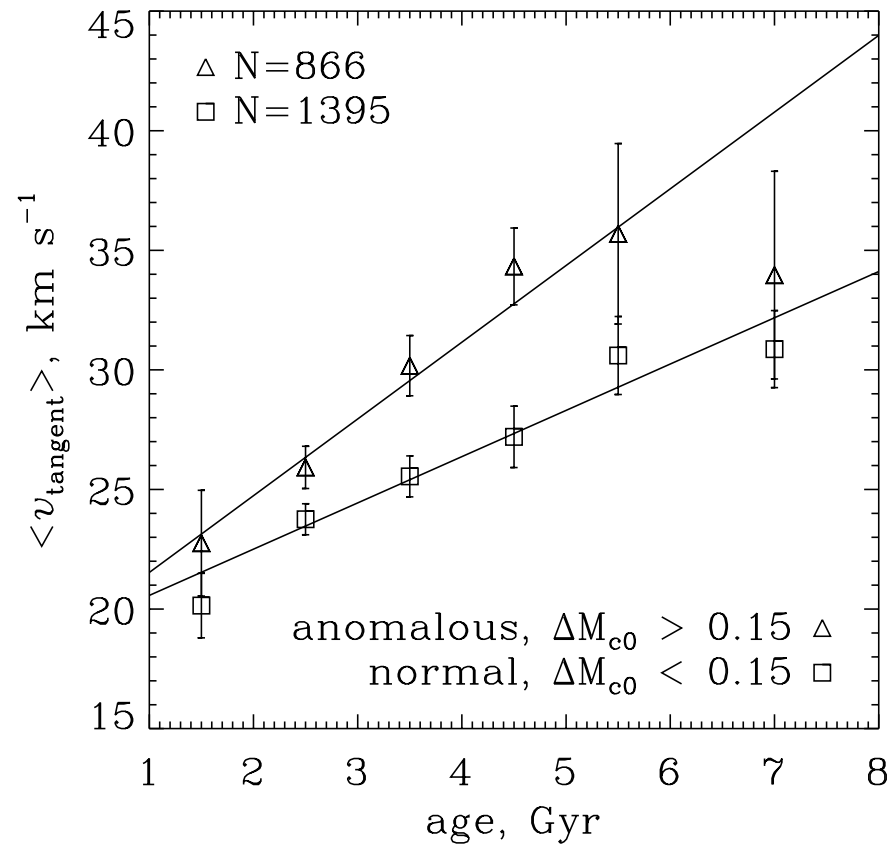

Fig. 7. Age-velocity relation (AVR) for the normal and anomalously bright $\mathrm{F}$ stars (see text for details)

the literature as related to the existence of blue stragglers and other unusual stars in stellar clusters (Pols \& Marinus 1994), apparent age discrepancy between the components in low mass binaries (Clausen et al. 1999), young age of the known unresolved binary stars (Suchkov 2000), etc. However, enhanced convective core mixing seems to be the only mechanism capable of extending the main sequence evolution by a significant amount.

It is to be noted that despite the potentially longer than normal lifetime, the actual average main sequence lifetime of the known unresolved binary stars is shorter than that of the single stars (Suchkov 2000). As hypothesized in the latter paper, this may be related to violent interaction of the binary's stellar components, which effectively removes the binary's primary in the colormagnitude diagram from the region occupied by the main sequence F stars; mechanisms of such an interaction were considered, for instance, in Pols \& Marinus (1994). On the other hand, $\mathrm{C}$ binaries were found to be indeed significantly older, on average, than the truly single stars, possibly indicating that many of $\mathrm{C}$ binaries have substellar secondaries incapable of causing too much damage to the primary $\mathrm{F}$ star.

Unidentified close binaries may represent a large fraction of the local population of $\mathrm{F}$ stars. Assuming that "single" F stars with $\Delta M_{c_{0}}>0.15$ are in fact tight binary pairs, the fraction of such binaries is at least $\sim 40 \%$. Thus, close binaries may heavily impact stellar population statistics used to probe the formation and evolution of the Galaxy. Therefore, these stars are important not only for better understanding of stellar evolution, but also for developing the adequate picture of the history of the Galaxy.
In conclusion, we can summarize our results as follows. (i). The unresolved binary $\mathrm{F}$ stars from the HIPPARCOS are typically much brighter than predicted on the basis of their uvby colors. (ii). This discrepancy, called here brightness anomaly, inversely correlates with the binary components separations, suggesting that at least part of it is associated with binary components interaction in tight pairs. (iii). Brightness anomaly correlates with stellar kinematics in a way that implies higher, on average, anomaly values at older ages. (iv). With ages from the same set of isochrones, the age-velocity relations of the normal single stars and binary candidates with brightness anomaly (C binaries) are discrepant in a way implying that the actual ages of $\mathrm{C}$ binaries are older than predicted.

These results argue that the main sequence evolution of the primary $\mathrm{F}$ star in a tight binary pair is different from that of a similar single star, evidently because of the interaction with the secondary. The difference appears to be consistent with extra mixing in the convective core of the primary, presumably induced by that interaction; however, other mechanisms need to be carefully studied before a definitive conclusion can be reached.

Acknowledgements. I would like to thank the anonymous referee for helpful comments and suggestions. It is my pleasure to thank Claus Leitherer, Stefano Casertano, and Nikolai Piskunov for stimulating discussions. I am grateful to S. Lyapustina and G. Galas for careful reading the paper and numerous revision suggestions.

\section{References}

Andersen, J., \& Nordström, B. 1999, in Theory and Tests of Convection in Stellar Structure (TTCSS), ASP Conf. Ser., 173, 31

Carlberg, R. G., Dawson, P. C., Hsu, T., \& VandenBerg, D. 1985, ApJ, 294, 674

Chiosi, C. 1999, in TTCSS, ASP Conf. Ser., 173, 9

Clausen, J. V., Baraffe, I., Claret, A., \& VandenBerg, D. A. 1999, in TTCSS, ASP Conf. Ser., 173, 265

Crawford, D. L. 1975, AJ, 80, 955

Demarque, P., Chaboyer, B., Guenther, D., et al. 1996, Yale Isochrones 1996 in "Sukyoung Yi's WWW Homepage"

Gimenez, A., Claret, A., Ribas, I., \& Jordi, C. 1999, in TTCSS, ASP Conf. Ser., 173, 41

Evans, D. W., van Leeuwen, F., Penston, M. J., Ramamani, N., \& Hog, E. 1992, A\&A, 258, 149

Griffin, R. F., \& Suchkov, A. A. 2001, in preparation

Hauck, B., \& Mermilliod, M. 1998, A\&AS, 129, 431

Mignard, F., Froschle, M., Badiali, M., et al. 1992, A\&A, 258, 165

Mignard, F., Froschle, M., \& Falin, J. L. 1992, A\&A, 258, 142

Moon, T. T. 1985, Commun. Univ. London Obs., No. 98

Moon, T. T., \& Dworetsky, M. M. 1985, MNRAS, 217, 305

Pols, O. R., \& Marinus, M. 1994, A\&A, 288, 475

Schuster, W. J., \& Nissen, P. E. 1989, A\&A, 221, 65

Söderhjelm, S., Evans, D. W., van Leeuwen, F., \& Lindegren, L. 1992, A\&A, 258, 157

Suchkov, A. A. 2000, ApJ, 535, L107

Suchkov, A. A., \& McMaster, M. 1999, ApJ, 524, L99 